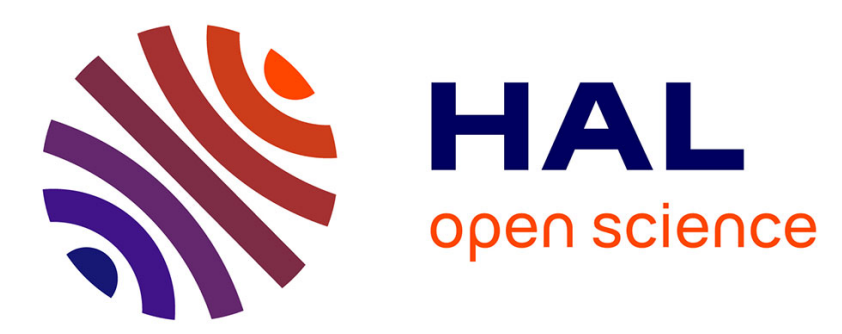

\title{
Subgap structure in the conductance of a three-terminal Josephson junction
}

\author{
Andreas H. Pfeffer, Jean Eudes Duvauchelle, Hervé Courtois, Régis Mélin, \\ Denis Feinberg, Francois Lefloch
}

\section{- To cite this version:}

Andreas H. Pfeffer, Jean Eudes Duvauchelle, Hervé Courtois, Régis Mélin, Denis Feinberg, et al.. Subgap structure in the conductance of a three-terminal Josephson junction. Physical Review B: Condensed Matter and Materials Physics (1998-2015), 2014, 90 (7), pp.075401. 10.1103/PhysRevB.90.075401 . cea-01054613

\section{HAL Id: cea-01054613 https://hal-cea.archives-ouvertes.fr/cea-01054613}

Submitted on 7 Aug 2014

HAL is a multi-disciplinary open access archive for the deposit and dissemination of scientific research documents, whether they are published or not. The documents may come from teaching and research institutions in France or abroad, or from public or private research centers.
L'archive ouverte pluridisciplinaire HAL, est destinée au dépôt et à la diffusion de documents scientifiques de niveau recherche, publiés ou non, émanant des établissements d'enseignement et de recherche français ou étrangers, des laboratoires publics ou privés. 


\title{
Subgap structure in the conductance of a three-terminal Josephson junction
}

\author{
A. H. Pfeffer,,${ }^{1,2}$ J. E. Duvauchelle, ${ }^{1,2}$ H. Courtois, ${ }^{3,4}$ R. Mélin, ${ }^{3,4}$ D. Feinberg,,${ }^{3,4}$ and F. Lefloch ${ }^{1,2, *}$ \\ ${ }^{1}$ Université Grenoble Alpes, INAC-SPSMS, F-38000 Grenoble, France \\ ${ }^{2}$ CEA, INAC-SPSMS, F-38000 Grenoble, France \\ ${ }^{3}$ Université Grenoble-Alpes, Institut Néel, F-38042 Grenoble, France \\ ${ }^{4}$ CNRS, Institut Néel, F-38042 Grenoble, France
}

(Received 19 July 2013; revised manuscript received 3 July 2014; published 4 August 2014)

\begin{abstract}
Three-terminal superconductor $(S)$-normal metal $(N)$-superconductor $(S)$ Josephson junctions are investigated. In a geometry where a T-shape normal metal is connected to three superconducting reservoirs, new subgap structures appear in the differential resistance for specific combinations of the superconductor chemical potentials. Those correspond to a correlated motion of Cooper pairs within the device that persist well above the Thouless energy and is consistent with the prediction of quartets formed by two entangled Cooper pairs. A simplified nonequilibrium Keldysh-Green's function calculation is presented that supports this interpretation.
\end{abstract}

DOI: 10.1103/PhysRevB.90.075401 PACS number(s): 74.78.Na, 74.45.+c, 74.50.+r, 85.25.Cp

\section{INTRODUCTION}

Josephson effects appear in superconductor-normal metalsuperconductor $(S N S)$ junctions where two superconductors $(S)$ are electrically coupled through a nonsuperconducting material $(N)$ [1]. The underlying mechanism is the Andreev reflection that converts a Cooper pair in $S$ into two phasecorrelated electrons in $N$ [2].

At zero voltage, the appearance of coherent Andreev bound states leads to a nondissipative supercurrent through the junction and a minigap in the density of states (DOS) of the normal metal. In the case of diffusive junctions and when the length of the normal part $L$ is longer than the superconducting phase-coherence length, both the supercurrent and the minigap scale with the Thouless energy given by $E_{\mathrm{Th}}=\hbar D / L^{2}$, where $D$ is the diffusive constant of the normal metal.

At finite voltages, the nonequilibrium subgap current is governed by multiple Andreev reflections (MARs). In this regime, MARs successively raise a quasiparticle's energy until it reaches the superconducting gap $\Delta$. Due to the superconductor density of states singularity at the gap edge, MARs lead to a subgap structure in the junction differential conductance for $e V=2 \Delta / n[3,4], n$ being an integer. This structure can be observed in diffusive $S N S$ junctions where the diffusion time through the junction is much smaller than the inelastic-scattering time.

In addition to this dc subgap quasiparticle transport, ac Josephson currents also appear in a diffusive $S N S$ junction. However, during the diffusion of an Andreev pair through the junction, phase coherence is maintained only if the energy of the electron or the hole compared to the superconductor chemical potential is smaller than the Thouless energy $E_{\mathrm{Th}}$ [5]. The ac Josephson current can be indirectly revealed under microwave irradiation. Shapiro steps [1] in the dc currentvoltage characteristics show up when the superconducting phase difference oscillation frequency $2 \mathrm{eV} / \hbar$ matches the microwave frequency or some multiple of it. The mere existence of Shapiro steps and therefore the ac Josephson currents essentially require a quasistatic superconducting

*Corresponding author: francois.lefloch@cea.fr phase difference, i.e., a diffusion time smaller than the inverse of the Josephson frequency $2 e V / \hbar$, or equivalently $e V<E_{\mathrm{Th}}$.

More recently, multiterminal junctions started to be investigated and brought a wealth of new properties, among which several remain to be experimentally uncovered. When two normal conductors are closely connected to a superconducting reservoir, crossed Andreev reflections (CARs) can inject two phase-correlated particles, one in each conductor, which amounts to split a Cooper pair into two entangled electrons [6-15]. This only occurs when the distance between the two normal conductors is smaller than the superconducting coherence length.

Another situation is met in mesoscopic three-terminal Josephson junctions in which a single normal conductor is connected to three superconducting contacts [16-20]. The transport properties then depend on two independent (phase or voltage) variables. Therefore, in addition to usual Josephson processes coupling two terminals, new mechanisms are expected that connect all three reservoirs. Several theoretical predictions have been made for such systems [16,17,21-23]. Nonlocal MARs should show up in the so-called incoherent MAR regime where the dwell time exceeds the coherence time [17,24]. On the other hand, the coherent regime where several MARs can occur within the coherence time is also very interesting. Shapiro-like resonances in the absence of external microwaves have been predicted whenever two ac Josephson frequencies match [21]. On similar grounds, the production of nonlocal quartets, as pairs of correlated Cooper pairs, has been proposed as a new dissipationless dc transport mechanism, which is phase coherent, despite the nonequilibrium conditions [22,23]. This present paper reports on an experimental study of such phenomena.

In this article, we report about electronic subgap transport in three-terminal Josephson junctions performed upon a piece of diffusive normal metal connected to three superconducting reservoirs. The junctions are all phase coherent as their length is smaller than the single-particle phase-coherence length $L_{\Phi}$ and in the long junction regime, e.g., the Thouless energy is much smaller than the superconducting gap. They are also rather symmetric and with a high transparency at every $S N$ interface leading to a large subgap Andreev current. Compared 
to a pair of two-terminal junctions, additional subgap structures are observed over a wide voltage range, well above the Thouless energy in a regime where one does not expect the presence of strong ac Josephson currents.

In the following, Sec. II contains the experimental details and reports the subgap anomalies. Section III is devoted to a physical discussion of the possible interpretations. Section IV concludes with perspectives.

\section{THE EXPERIMENT}

\section{A. Samples and measurement process}

The samples we have studied have been fabricated by a shadow mask evaporation technique [see scanning electron microscope (SEM) images in Figs. 1 and 2]. Copper and aluminum were evaporated at different angles through a polymethylmethacrylate/methacrylic acid bilayer mask in an ultrahigh vacuum chamber. The evaporation of a thin $\mathrm{Cu}$ layer of 50-nm thickness was followed immediately by the evaporation of thick Al electrodes of thickness $500 \mathrm{~nm}$ without breaking the vacuum, leading to highly transparent and uniform $S N$ interfaces. The width of the normal metal is about $0.6 \mu \mathrm{m}$, and its length $L$ is around $1 \mu \mathrm{m}$. Using a diffusion constant for copper $D=100 \mathrm{~cm}^{2} / \mathrm{s}$, we get a Thouless energy $E_{\mathrm{Th}}=\hbar D / L^{2} \simeq 6 \mu \mathrm{eV}$. This value is confirmed by fitting the temperature dependence of the critical current between two of the superconducting contacts [25]. The superconducting aluminum energy gap is $\Delta=170 \mu \mathrm{eV}$ [26]. The diffusion time $\tau_{D}=L^{2} / D \simeq 0.1 \mathrm{~ns}$ is much smaller than the inelastic time $\tau_{\text {in }} \simeq 1 \mathrm{~ns}$ at $100 \mathrm{mK}$.

Three-terminal differential resistances were measured using an experimental setup specially designed to perform highly sensitive measurements of current average and fluctuations in low-impedance nanodevices at very low temperatures [27], see Fig. 1. The experiment operates down to $30 \mathrm{mK}$ and is equipped with three commercial superconducting quantum interference devices (SQUIDs) as current amplifiers. Each

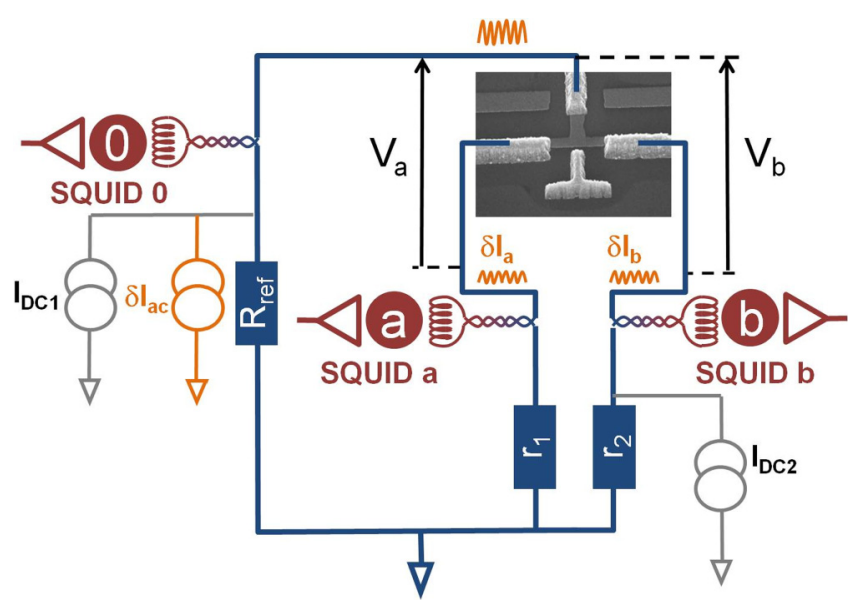

FIG. 1. (Color online) Experimental setup for differential resistance measurements [27]. The three macroscopic resistors have low-resistance values $(\simeq 0.1 \Omega)$ allowing voltage biasing the samples. The SEM image shows a three-terminal junction sample with a T-shape geometry. device terminal is connected to the input coil of a SQUID in series with a macroscopic resistor with a low resistance $R_{\text {ref }} \simeq r_{1} \simeq r_{2} \simeq 0.09 \Omega$.

The measurement scheme consists of sending an ac current modulation $\delta I_{\mathrm{ac}}=1 \mu \mathrm{A}$ on the reference side and recording the current in each branch of the circuit. The differential resistance $R_{\text {diff, } a(b)}$ then reads

$$
R_{\text {diff }, a(b)}=R_{\text {ref }}\left(\delta I_{\mathrm{ac}}-\delta I_{0}\right) / \delta I_{a(b)}-r_{1(2)},
$$

where $\delta I_{i}$ is the ac current measured in the SQUID and $i=0, a$, or $b$. For all the samples studied here, $R_{\mathrm{diff}, a}$ and $R_{\text {diff, } b}$ give the same behavior. In order to explore the nonlinear response in the $\left(V_{a}, V_{b}\right)$ plane, two dc current sources were used, and the voltage differences $V_{a}$ and $V_{b}$ were measured with two room-temperature differential voltage amplifiers. In practice, $I_{\mathrm{DC} 2}$ is first set to a fixed value, and $I_{\mathrm{DC} 1}$ is ramped with current steps of 1 or $2 \mu \mathrm{A}$. When the ramp is finished, $I_{\mathrm{DC} 2}$ is increased by a larger current step (typically $20 \mu \mathrm{A}$ ), and $I_{\mathrm{DC} 1}$ is ramped again. The density of the measurement points is therefore not uniform, which explains the dotted features observed in the contour plots.

\section{B. Results}

Figure 2 shows the experimental data measured at $T=$ $100 \mathrm{mK}$ in a sample with separated junctions (see SEM image in Fig. 2). For this sample geometry, the separation holds as the $\mathrm{Cu}$ underneath the central electrode, although being continuous, is thin enough that the locally induced gap is that of the superconducting gap $\Delta$ of aluminum. Only two anomalies corresponding to dc Josephson effects at $V_{a}=0$ and $V_{b}=$ 0 are detected. This confirms the absence of multiterminal effects in the presence of a central electrode with a width ( $\sim 900 \mathrm{~nm}$ ) larger than the superconducting coherence length $\xi_{s}$ as already reported [18]. Such a device therefore behaves like two independent $S N S$ junctions in parallel.

Here and in the following, the voltage range was limited to $\pm 40 \mu \mathrm{V}$ because going beyond would require a dc current close to the superconducting electrodes depairing current [18]. As the investigated voltage range remains well below the

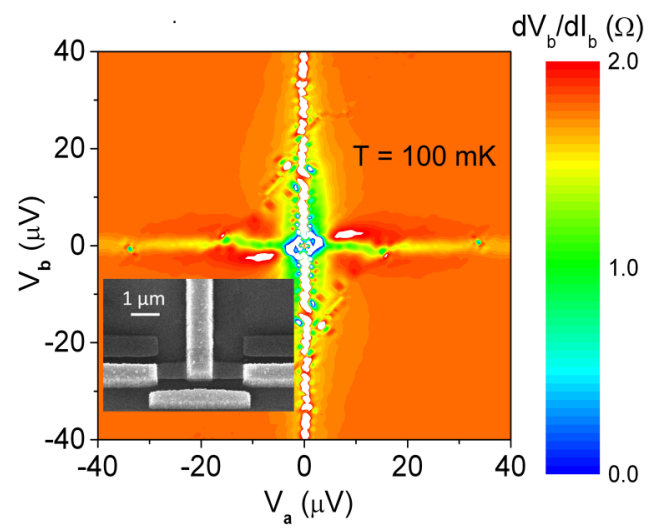

FIG. 2. (Color online) Differential resistance $R_{\mathrm{diff}, b}$ of a threeterminal device with separated normal metal parts in the $\left(V_{a}, V_{b}\right)$ plane at $T=100 \mathrm{mK}$. The SEM image represents a sample with such a typical geometry. In this case, only the upper half with $V_{b}>0$ has been measured, and the graph has been symmetrized. 

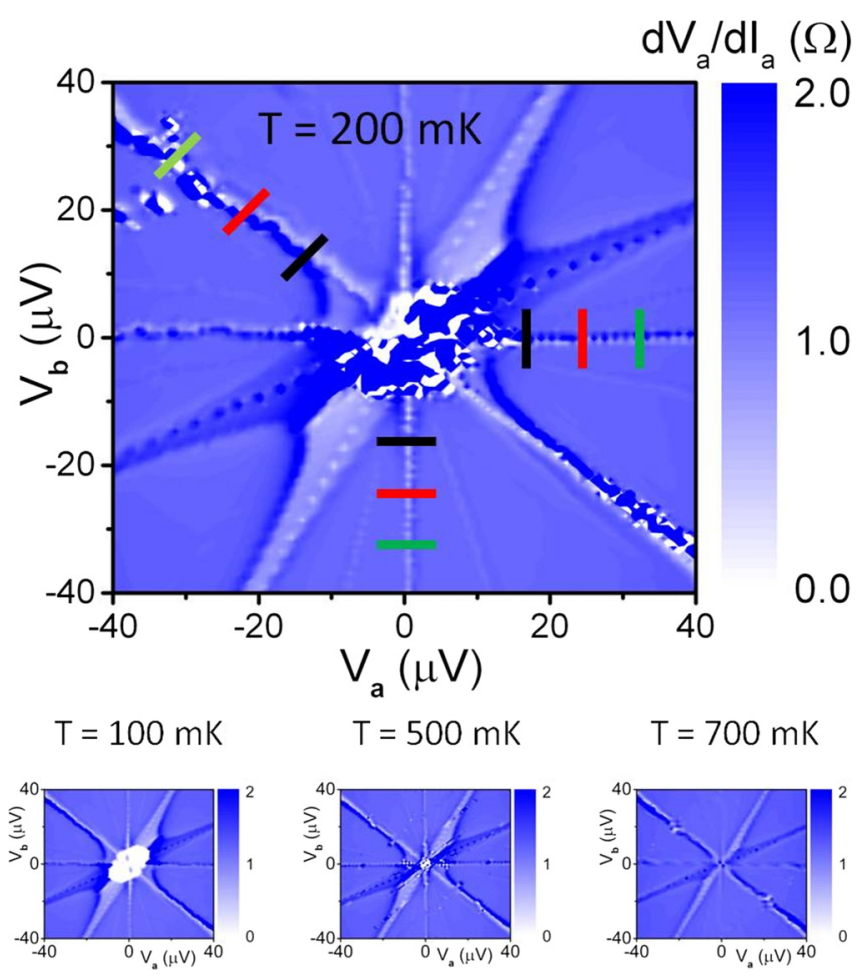

FIG. 3. (Color online) Differential resistance $R_{\text {diff, } a}$ of a T-shape junction in the $\left(V_{a}, V_{b}\right)$ plane for various temperatures. At $T=$ $200 \mathrm{mK}$, the data have been measured for the entire voltage range. For the other temperatures, only the upper half with $V_{b}>0$ has been measured, and the graph has been symmetrized for clarity.

superconductor energy gap, the number of multiple Andreev reflections $(\sim 2 \Delta / e V)$ necessary for a quasiparticle to reach the superconducting gap is more than 8 , which would correspond to a total diffusion time much larger than the inelasticscattering time. This defines a strong interaction regime in which MAR cycles are interrupted by inelastic events. In such a bath of thermalized hot quasiparticles carrying an elevated effective temperature, the MAR-induced steps in the energy distribution function are completely washed out [26,28], and hence the subgap structures related to the singularity of the DOS at the $S / N$ interface cannot be observed.

We have investigated another type of three-terminal junction with a T-shape normal conductor connecting three superconducting electrodes $S_{0}, S_{a}$, and $S_{b}$ whose SEM image is shown in Fig. 1. Here, $S_{0}$ corresponds to the upper central superconducting electrode, and $S_{a}$ and $S_{b}$ correspond to the left and right superconducting electrodes, respectively. The differential resistance $R_{\text {diff, } a}$ is shown in Fig. 3 at $T=200 \mathrm{mK}$. For this geometry, we expect three Josephson couplings $J_{0 a}, J_{0 b}$, and $J_{a b}$ where the two indices label the two involved superconducting terminals. In Fig. 3, the couplings $J_{0 a}$ and $J_{0 b}$ are clearly observed at $V_{a}=0$ and $V_{b}=0$, respectively. As expected from the definition of the differential resistance [Eq. (1)], the Josephson coupling $J_{0 a}$ appears as a dip in the differential resistance $R_{\text {diff, } a}$, whereas $J_{0 b}$ shows up as a peak. We have checked that the opposite behavior is observed when plotting $R_{\text {diff, } b}$.
We can see in Fig. 3 that the coupling $J_{a b}$ does not show up at $V_{b}-V_{a}=0$. In the actual experiment, the ac modulation was sent to the central electrode $S_{0}$ so that the separation of this current into the two branches $S_{a}$ and $S_{b}$ is not sensitive to the coupling $J_{a b}$. We have verified in a similar sample that the latter coupling is indeed revealed when sending the ac modulation through $S_{a}$ or $S_{b}$.

In addition to the two dc Josephson features discussed above, three other lines are clearly visible at $V_{b}=-V_{a}, V_{b}=$ $2 V_{a}$, and $V_{b}=1 / 2 V_{a}$. Notice that in a T-shape geometry, the three superconducting reservoirs are equivalent, meaning that the voltages $V_{a}, V_{b}$, and $V_{a}-V_{b}$ are also equivalent. We can thus state that these three lines all originate from the same type of mechanism involving the three superconducting contacts. The observation of this subgap structure in the low-bias differential conductance of a three-terminal superconducting hybrid device is the main experimental finding of the present paper.

In a second step, we have studied the temperature dependence of the differential resistance of the T-shape device. The results are plotted at the bottom of Fig. 3. Apart from the central part that is related to the dc Josephson effect at very low bias, the subgap structure does not evolve much with temperature. All lines are found to be clearly visible up to $700 \mathrm{mK}$ and $40 \mu \mathrm{V}$. This confirms that, in the voltage range under investigation, the electronic temperature is well above the bath temperature [29].

To further investigate these new features, we have plotted some line traces perpendicular to the $V_{b}=-V_{a}$ line [Fig. 4(a)], to the $V_{a}=0$ line [Fig. 4(b)], and to the $V_{b}=0$ line [Fig. 4(c)] for various levels of the applied voltages as indicated by the colored lines in Fig. 3. As expected, the differential resistance $R_{\text {diff, } a}$ appears as a dc Josephson resonance around $V_{a}=0$ for any value of $V_{b}$ [Fig. 4(b)]. The same type of response is observed when plotting $R_{\mathrm{diff}, b}$ around $V_{b}=0$

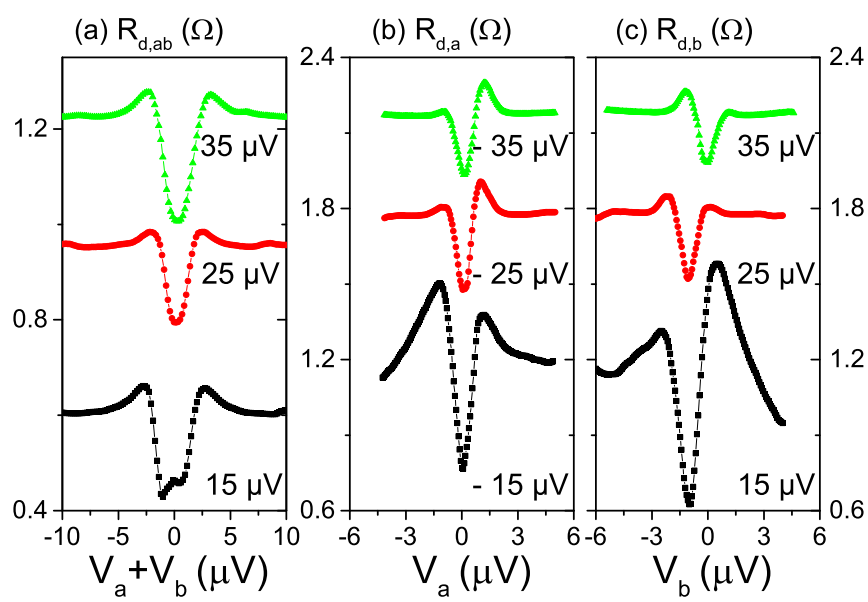

FIG. 4. (Color online) Line traces at various values of the applied voltage of (a) the differential resistance of the full sample considering the two branches $a, b$ as being in parallel as a function of the voltage $V_{a}+V_{b}$ for several values of $\left(V_{a}-V_{b}\right) / 2$, (b) the differential resistance of branch $a$ vs $V_{a}$ for various values of $V_{b}$, and (c) the differential resistance of branch $b$ vs $V_{b}$ for various values of $V_{a}$. The color code follows that of the lines in Fig. 3. The data are shifted for clarity except for the lower ones. 
for any value of $V_{a}$ [Fig. 4(c)]. It turns out that when plotting the overall sample differential resistance recalculated by considering the two branches $a$ and $b$ as being in parallel [ $\left.R_{\text {diff, } a b}=R_{\text {diff, } a} R_{\text {diff }, b} /\left(R_{\text {diff, } a}+R_{\text {diff }, b}\right)\right]$ as a function of the voltage $V_{a}+V_{b}$, the observed profile of the subgap structure across the $V_{b}=-V_{a}$ line is also in striking resemblance to a Josephson resonance. This observation suggests that the additional anomalies are due to coherent effects involving the three terminals.

Moreover, it is important to notice that the features discussed here are rather robust and constant with respect to the applied voltage. More precisely, as seen in Fig. 4, those persist at energies well above the Thouless energy. Therefore, the scheme to explain the additional features seen at nonzero $V_{a}$ and $V_{b}$ and that involve the three terminals must also be robust against voltage-induced dephasing towards all the branches of the device.

\section{INTERPRETATION}

\section{A. Synchronization of ac Josephson effects}

Subgap structures similar to the ones observed here were predicted and observed in the conductance of coupled but separated junctions [30,31]. In this case, two ac Josephson currents coexist with frequencies $v_{a}=2 e V_{a} / h$ and $v_{b}=$ $2 e V_{b} / h$. When the two frequencies match, e.g., $V_{a}= \pm V_{b}$, down-mixing through the nonlinear response of the device can generate dc subgap structures similar to Shapiro steps. In our experimental scheme with a low-resistive environment ( $R_{\text {ref }}, r_{1}, r_{2} \ll R_{n}$ ), this coupling could be obtained through the external circuit. Yet, no anomalies are observed in the sample with separate junctions, despite the fact that both samples have exactly the same circuit environment. In fact, due to the SQUID inductances and the wiring, the external impedances at the Josephson frequency are much larger than the resistances of the bias resistors, preventing any ac Josephson current to circulate in the external circuit. Therefore, the relevant coupling can only be within the sample itself.

An extended resistively shunted junction (RSJ) model generalizing that of Ref. [30] could provide a phenomenological description. It involves a triangular Josephson array, shunted by the corresponding normal-state resistances, that account for the quasiparticle processes within the $N$ region. With such a model, the observation of strong resonances requires sizable ac Josephson currents, whereas they are known to decrease when the voltages $e V_{a, b}$ exceed the Thouless energy $E_{\mathrm{Th}}$ [32]. Thus, even if the voltage decrease in ac Josephson currents is expected to be progressive, it should definitively lead to a reduction in the resonance for such a variation in $V_{a(b)}$. This is very much in contrast to what is observed in Fig. 4.

In addition to be quantitatively inconsistent, such an RSJ model is only phenomenological. Due to the long coherence time, transport in the present experimental conditions is truly mesoscopic, and the explanation of the observations requires a phase-coherent microscopic mechanism taking place in the normal region.

One might consider a more microscopic approach and seek how the possible ac Josephson oscillations can synchronize together to yield a constant dc component. Such a problem indeed is similar to the one considered in Refs. [25,33,34]. In a clean $S N S$ junction polarized with a voltage $V$, oscillations with a frequency double of the basic Josephson frequency $\omega=$ $\frac{2 \mathrm{eV}}{\hbar}$ are generated and show up under microwave irradiation as half-integer Shapiro steps. This was explained by Argaman [33] within a semiphenomenological description in which the both the Andreev levels and their steady-state distributions oscillate at the Josephson frequency. The argument applies in the adiabatic regime in which the Thouless energy is much larger than the applied voltage.

The same argument could be applied to our three-terminal Josephson junctions for which the oscillating ac Josephson current at a frequency $v_{a}$ between two of the three terminals could be modulated by oscillation of the distribution function due to the Josephson coupling between two other terminals at a frequency $v_{b}$. In that case, the second-harmonic response obtained by Argaman [33] in the case of a two-terminal $S N S$ junction transposes into a response at a frequency $v_{a}+v_{b}$ giving rise to dc features when $v_{a}=-v_{b}$.

Again, the conditions for such a scenario to apply are the same as in Refs. [25,33,34], e.g., that the voltage is small enough to allow an adiabatic approximation both in the current components and in the Andreev state distribution. The first one requires that $e V \ll E_{\mathrm{Th}}$, the minigap scale, and the second is even more restrictive, $e V \ll \sqrt{E_{\mathrm{Th}} \frac{\hbar}{\tau_{\mathrm{in}}}}$. For instance, in the experiment of Lehnert et al. [34], the frequency doubling is observed for $e V<40 \mu \mathrm{eV}$, an order of magnitude below the Thouless energy $E_{\mathrm{Th}}=350 \mu \mathrm{eV}$. But in the experiment reported in our paper, it is the other way around! The subgap anomalies are observed for $e V$ above $E_{\mathrm{Th}}$, up to $8 E_{\mathrm{Th}}$, only limited by experimental constraints and without any sign of decay. Thus, although qualitatively appealing, the above mechanism does not provide a good explanation for our experimental results.

\section{B. The quartet scenario \\ 1. Qualitative description}

The limitation of the synchronization scenario is the voltage-induced dephasing suffered by the two electrons of each of the Cooper pairs transferred between two superconducting terminals. Let us instead show that the quartet mechanism, proposed for clean bijunctions [22,23], can be generalized to a diffusive system and is fully robust against dephasing at voltages much higher than $E_{\mathrm{Th}}$.

The main idea is that two Cooper pairs are transferred in a single and fully energy-conserving quantum process in which the two pairs cross in an entangled way by exchanging an electron between them.

To describe this mechanism, let us consider a piece of diffusive normal metal $N$ connected to three superconducting reservoirs $\left(S_{0}, S_{a}\right.$, and $\left.S_{b}\right)$ whose potentials are set to $V_{0}=$ $0, V_{a}=+V$, and $V_{b}=-V$ respectively, as depicted in Fig. 5 . Two Cooper pairs from $S_{0}$ can be simultaneous split in $N$, each of them in two electrons with opposite energies (with respect to the Fermi energy) that we define, without losing generality, as $\pm(e V+\varepsilon)$ and $\pm(e V-\varepsilon)$. When these energies are larger than the Thouless energy $E_{\mathrm{Th}}$, the two electrons of each pair do not follow the same trajectory. Nevertheless, if the energy $\varepsilon$ 


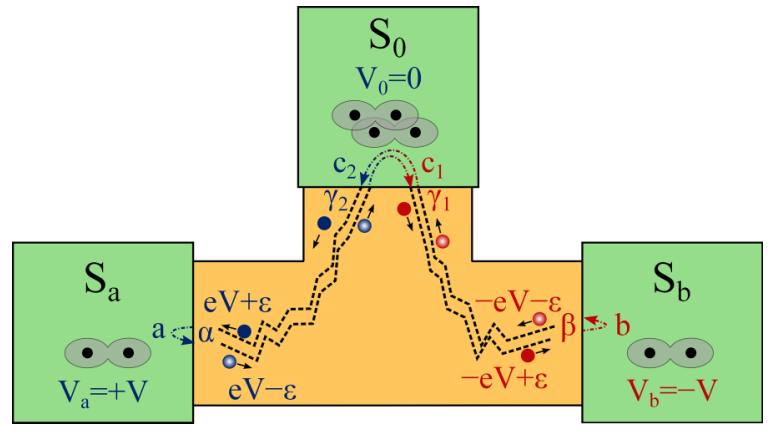

FIG. 5. (Color online) Schematic for $Q_{0}$ quartet production from $S_{0}$ to $S_{a}$ and $S_{b}$. Two Cooper pairs are split simultaneously at $S_{0}$ with one electron of each pair propagating towards $S_{a}$ and $S_{b}$ where, under the appropriate energy condition $\left(V_{a}=-V_{b}\right)$, they recombine to create two separated Cooper pairs.

is small compared to $E_{\mathrm{Th}}$, the electron of the first pair at $e V+\varepsilon$ can follow the same phase-coherent trajectory as the electron of the second pair at $e V-\varepsilon$ and, for instance, reach $S_{a}$. Since $V_{a}=+V$, the two particles have relative opposite energies $\pm \varepsilon$ and can recombine as a Cooper pair in $S_{a}$. The same mechanism holds for the two other particles at $S_{b}$. In the whole process, two Cooper pairs from $S_{0}$ are split altogether to create two spatially separated Cooper pairs in $S_{a}$ and $S_{b}$, a so-called quartet, named hereafter $Q_{0}$ as it originates from $S_{0}$.

The key point favoring the quartet mechanism is that the coherence of each Andreev pair reaching $S_{a}$ or $S_{b}$ can be satisfied at any voltage $V_{a}=-V_{b}$, even when $\left|e V_{a(b)}\right|>E_{\mathrm{Th}}$. Considering again the four electrons emitted from the two split pairs, two of them have energies $e V \pm \varepsilon$ [pair (a),] and the two others have energies $e V \pm \varepsilon$ [pair (b)] (see Fig. 5). As the quartet mechanism is a quantum process, the sum of all the possible diffusion probabilities has to be considered altogether. Among those, the situation where pair (a) propagates towards $S_{a}$ and pair $(b)$ towards $S_{b}$ is phase coherent and independent of the applied voltage $V$. Indeed, the phase difference accumulated by pair $(a)$ [pair $(b)$ ] scales as $\varepsilon \tau_{D a(b)} / \hbar$ where $\tau_{D a(b)}$ is the diffusion time from $S_{0}$ to $S_{a}\left(S_{b}\right)$. The quartet mode is therefore a fully coherent dc process taking place in the mesoscopic $N$ region and involving four Andreev reflections.

This is very different from the scheme where two electrons of a single Cooper pair propagate towards $S_{a}$ or $S_{b}$. In that case, the two electrons of a single pair have energies $\pm(e V+\varepsilon)$ or $\pm(e V-\varepsilon)$, and the accumulated phase difference scales with $e V / E_{\mathrm{Th}}$. The effect of such trajectories has, therefore, a vanishing contribution to the electronic transport when $e V \gg$ $E_{\mathrm{Th}}$.

Let us note that the quartet response bears some resemblance to MARs [17] with two important differences. First, the total energy balance of the process is zero, and second, it does not lead to quasiparticle transport above the superconducting gap.

In this quartet description, the line at $V_{b}=-V_{a}$ corresponds to the production of quartets $Q_{0}$, whereas the line at $V_{b}=2 V_{a}\left(V_{b}=1 / 2 V_{a}\right)$ originates from quartets $Q_{a}\left(Q_{b}\right)$ produced in $S_{a}\left(S_{b}\right)$ towards $S_{0}$ and $S_{b}\left(S_{0}\right.$ and $\left.S_{a}\right)$. At lowest order, the quartet mechanism requires only four Andreev reflections, much less than needed in the same voltage range for a quasiparticle to reach the gap edge in a usual MAR process, which makes the quartet mechanism much more robust to inelastic collisions.

\section{Sketch of the microscopic calculation}

The above arguments can be substantiated by a microscopic calculation (the Appendix), valid under the separation of energy scales $E_{\mathrm{Th}}<e V<\Delta$. One uses nonequilibrium Keldysh-Green's functions and performs a lowest-order calculation in tunnel amplitudes at the different $S N$ interfaces, inspired by Ref. [35]. The quartet current is calculated using a Hamiltonian formalism, and an essential step is averaging over disorder.

The current appears as a sum of contributions, each being a product of six propagating amplitudes associated with the diagrammatic lines in Fig. 5 [Eqs. (A7)-(A9)]. As a classical procedure in the treatment of diffusion in metals and in an $S N S$ junction [36], disorder averaging takes advantage of the energy separation of these lines. In fact, two lines are correlated by disorder provided their energies are closer than $E_{\mathrm{Th}}$. It results that the averaged product of six amplitudes can be decoupled into three factors. One corresponds to the diffusion of an Andreev pair from $S_{0}$ to $S_{a}$ at energies $e V \pm \varepsilon$ with $\varepsilon<E_{\mathrm{Th}}<e V$, another one corresponds to the diffusion of an Andreev pair from $S_{0}$ to $S_{b}$ at energies $-e V \pm \varepsilon$, and the third corresponds to the anomalous diffusion within $S_{0}$ that achieves Andreev reflection at energies $e V$ and $-e V$ [37].

The principle of the above calculation can be benchmarked on the simpler case of an $S N S$ junction at equilibrium for which the coherent pair current is proportional to the singleparticle conductance $G_{N}$ times the coherent energy window given by the Thouless energy. This leads to the known scaling for the critical current $e I_{c} \propto G_{N} E_{\mathrm{Th}}$ [25].

The main result of our calculation detailed in the Appendix is to show that the coherent quartet current has a similar form and is given by the two-particle CAR conductance times the same energy window $E_{\mathrm{Th}}$. It follows a scaling given by

$$
e I_{Q} \sim-G_{\mathrm{CAR}} E_{\mathrm{Th}} .
$$

The minus sign comes from the exchange and recombination process [23]. The conductance $G_{\mathrm{CAR}}$ is the crossed Andreev conductance of a $N_{a} N S_{0} N N_{b}$ structure in which the electrodes $S_{a}$ and $S_{b}$ are in the normal state and at voltages $\pm V$.

The CAR conductance can then be evaluated (see the Appendix) and recast as

$$
G_{\mathrm{CAR}} \sim \frac{G_{N_{a}} G_{N_{b}}}{G_{0}\left(\xi_{s}\right)},
$$

where $G_{N_{a, b}}$ is the conductance within each normal branch of the bijunction and $G_{0}\left(\xi_{s}\right)$ is the normal-state conductance of a region of size $\xi$ of the superconductor $S_{0}$. This calculation shows that the ratio between the quartet maximum current at a bias $V$ and the single junction critical current at zero bias is $I_{Q} / I_{c}(0) \sim G_{\mathrm{CAR}} / G_{N} \sim G_{N} / G_{0}\left(\xi_{s}\right)$, which is not necessarily small. Based on measured sample parameters, we estimate this ratio to $\sim 0.1-0.5$, in fair agreement with the experiment. Notice that if $e V \ll \Delta, G_{\mathrm{CAR}}$ thus $I_{Q \text {,max }}$ does not decrease with $V$, in agreement with the present experiment. 


\section{CONCLUSION}

In conclusion, we reported about new subgap structures in the differential conductance of a metallic nanostructure with three superconducting reservoirs, a so-called bijunction. The existence of such anomalies well above the Thouless energy points towards a new and fully coherent mechanism, different from the synchronization of separated Josephson junctions, or any mesoscopic generalization of such a process. Our results are consistent with the production of nonlocal quartets as a resonant pair of Cooper pairs splitting and recombining within the $N$ region. Therefore, our results provide convincing experimental evidence for (double) crossed Andreev reflections in metallic superconducting/normal metal hybrid three-terminal nanostructures with a signature in the electronic response at low temperatures much larger than in metallic Cooper pair splitters using only one superconducting reservoir.

The quartet mechanism carries intrinsic four-particle entanglement, generalizing two-fermion entanglement from CAR's [10] that could be exploited if adding more degrees of freedom, such as in quantum dots [22]. More refined probes are necessary to quantitatively study the correlated pair transport involving quartets as well as possible other regimes not evidenced in the present experiments, such as the low-voltage adiabatic transport. A useful tool is to couple the bijunction to microwaves and to study the Shapiro steps coming from deviations from the resonant situation $V_{a}=-V_{b}=V$.

\section{ACKNOWLEDGMENTS}

This work has been partially funded by the French Research National Agency, ANR-NanoQuartet (Grant No. ANR12BS1000701). We acknowledge the Nanoscience Foundation for the Ph.D. grant of A. H. Pfeffer, the NanoFab facility at Institut Néel-CNRS for sample fabrication, and fruitful discussions with B. Douçot and C. Padurariu.

\section{APPENDIX: ANALYTICAL CALCULATION OF THE QUARTET CURRENT}

The superconductors $S_{0, a, b}$ are described by the mean-field BCS Hamiltonian with identical gaps $\Delta$ and phases $\varphi_{0}=$ $0, \varphi_{a}, \varphi_{b}$. To simplify, all materials $S_{i}, N$ are taken with the same bandwidth $w$, and they are connected by a hopping parameter $\tau$, related to the interface transparency $T$ by $T=\frac{4 \tau^{2} / w^{2}}{\left(1+\tau^{2} / w^{2}\right)^{2}}$. In Nambu notations, the hopping amplitudes take the form ( $i=0, a, b$, and $\alpha$ denoting the position on the interface)

$$
\hat{\Sigma}_{i, \alpha}(t)=\tau\left(\begin{array}{cc}
e^{i e V_{i} t} & 0 \\
0 & -e^{-i e V_{i} t}
\end{array}\right) .
$$

The local advanced Green's functions in the superconductors are as follows in the frequency domain $\left(\omega_{\eta}=\omega-i \eta\right)$ :

$$
\hat{g}_{i, i}^{A}(\omega)=\frac{1}{w \sqrt{\Delta^{2}-\omega_{\eta}^{2}}}\left(\begin{array}{cc}
-\omega_{\eta} & \Delta e^{i \varphi_{i}} \\
\Delta e^{-i \varphi_{i}} & -\omega_{\eta}
\end{array}\right),
$$

The retarded Green's functions are obtained by changing $\eta$ into $-\eta$ in the above expression. The choice of the gauge is such that the time dependence of the phases $2 e V_{a(b)} t / \hbar$ are included in the Nambu hopping amplitudes $\hat{\Sigma}_{a(b)}$ (with $\hbar=1)$. The phases $\varphi_{a(b)}$ at the origin of time are included in the off-diagonal components of the Nambu-Green's functions. The local advanced Green's functions are $\hat{g}^{A}(\omega) \sim i \pi \rho_{N}$ in the normal metal, where $\rho_{N}$ is the local density of states of the normal metal.

The current at some point $a$ of the interface of the superconductor $S_{a}$ is given by

$$
I_{a}(t)=\frac{2 e}{h} \mathcal{R} e\left[\hat{\Sigma}_{a, \alpha}(t) \hat{G}_{\alpha, a}^{( \pm), 11}(t, t)-\hat{\Sigma}_{a, \alpha}(t) \hat{G}_{\alpha, a}^{( \pm), 22}(t, t)\right]
$$

where $\hat{G}_{\alpha, a}^{( \pm), 11}(t, t)$ [respectively, $\hat{G}_{\alpha, a}^{( \pm), 22}(t, t)$ ] is the electron (respectively, hole) Keldysh-Green's function at point $a$.

Together with $\hat{G}^{(R, A)}, \hat{G}_{\alpha, a}^{( \pm)}(t, t)$ obeys a Dyson equation which allows calculating the current as a product of Green's functions propagating electrons (holes) in the normal or superconducting regions and hopping self-energies $\hat{\Sigma}$ at the interfaces. Stationarity allows Fourier transforming the time quantities and calculating the current contributions as a sum over the Fourier components $\hat{G}\left(\omega_{n}\right)$ with $\omega_{n}=$ $\omega+n e V$. Specifying to the voltages $V_{a}=V, V_{b}=-V$, the self-energies $\hat{\Sigma}(\omega)$ connect Green's functions with indices $n$ differing by \pm 1 .

The quartet diagram on Fig. 5 takes a typical chain form, starting at the $S_{0}-N$ interface (with the frequency arguments omitted),

$$
\begin{aligned}
(\mathcal{A})_{Q}= & \Sigma_{c_{1}, \gamma_{1}}^{11 / 00} g_{\gamma_{1}, \beta}^{11 / 00} \Sigma_{\beta, b}^{11 / 01} g_{b b}^{12 / 11} \Sigma_{b, \beta}^{22 / 12} g_{\beta, \gamma_{1}}^{22 / 22} \\
& \times \Sigma_{\gamma_{1}, c_{1}}^{22 / 22} g_{c_{1}, c_{2}}^{21 / 22} \Sigma_{c_{2}, \gamma_{2}}^{11 / 22} g_{\gamma_{2}, \alpha}^{11 / 22} \Sigma_{\alpha, a}^{11 / 21} g_{a a}^{12 / 11} \\
& \times \Sigma_{a, \alpha}^{22 / 10} g_{\alpha, \gamma_{2}}^{22 / 00} \Sigma_{\gamma_{2}, c_{2}}^{22 / 00} g_{0_{2}, c_{1}}^{21 / 00} .
\end{aligned}
$$

The first two upper labels correspond to Nambu matrix notation, and the second two correspond to the harmonics $\left(n, n^{\prime}\right)$ of half the Josephson frequency $\omega_{0}=\frac{2 e V}{\hbar}$. The advanced, retarded, and Keldysh labels have to be inserted in this expression, resulting in eight different terms. Next, each of the eight terms is evaluated. The final expression for $(\mathcal{A})_{Q}$ is as follows:

$$
\begin{aligned}
(\mathcal{A})_{Q}= & n_{F}(\omega-e V) \tau^{8} \\
& \times\left\{g_{a a}^{A} g_{\alpha, \gamma_{2}}^{A} g_{\gamma_{2}, \gamma_{1}}^{A} g_{\gamma_{1}, \beta}^{A} g_{b b}^{A} g_{\beta, \gamma_{1}}^{A} g_{\gamma_{1}, \gamma_{2}}^{A} g_{\gamma_{2}, \alpha}^{A}-A \leftrightarrow R\right\},
\end{aligned}
$$

where $A \leftrightarrow R$ means that advanced and retarded have been interchanged. The unperturbed Green's functions $g_{i j}^{A}$ represent the amplitudes for electron and hole propagation, and they are evaluated at the appropriate energies $\pm e V \pm \varepsilon$ shown in Fig. 5. Those energies correspond to the transitions between $n$ and $n^{\prime}$ indices [see Eq. (A4)] induced by the hopping matrix elements. A summation over the labels $\alpha(\beta)$ and $\gamma$ at the interfaces has to be carried out. This procedure is justified to describe extended contacts at lowest order in the tunnel amplitudes. As far as the applied voltages are small enough compared to the gap, the energy dependence of the Green's functions can be discarded.

The next step is to perform disorder averaging. A contribution, such as $(\mathcal{A})_{Q}$ should be replaced by its average $\left\langle\left\langle(\mathcal{A})_{Q}\right\rangle\right\rangle$ over disorder in the $N$ region and in the superconductors. Expression (A5) contains several amplitudes, matrices in $S_{i}$ and numbers in $N$. First, $g_{a a}^{A}, g_{b b}^{A}$, which yields density of 
states factors in $S_{a}$ and $S_{b}$. Second, the product $\left(g_{\gamma_{2}, \gamma_{1}}^{A} g_{\gamma_{1}, \gamma_{2}}^{A}\right)$ of amplitudes in $S_{0}$ at energies close to $e V$ (electrons) and $-e V$ (holes) that can be averaged separately. It describes the anomalous diffusion of a quasiparticle within $S_{0}$, yielding Andreev reflection at the $N S_{0}$ interface [37], and third, the product $\left(g_{\alpha, \gamma_{2}}^{A} g_{\gamma_{2}, \alpha}^{A} g_{\gamma_{1}, \beta}^{A} g_{\beta, \gamma_{1}}^{A}\right)$ of amplitudes in $N$. The two first amplitudes contribute at energies $e V \pm \varepsilon$, and the two others contribute at energies $-e V \pm \varepsilon$. We assume that $e V>E_{\mathrm{Th}}>\varepsilon$ and use the fact that the coherence between electron and hole trajectories is limited by the Thouless energy. Then it is justified to decouple $\left\langle\left\langle g_{\alpha, \gamma_{2}}^{A} g_{\gamma_{1}, \beta}^{A} g_{\beta, \gamma_{1}}^{A} g_{\gamma_{2}, \alpha}^{A}\right\rangle\right\rangle \simeq$ $\left\langle\left\langle g_{\alpha, \gamma_{2}}^{A} g_{\gamma_{2}, \alpha}^{A}\right\rangle\right\rangle\left\langle\left\langle\left(g_{\gamma_{1}, \beta}^{A} g_{\beta, \gamma_{1}}^{A}\right\rangle\right\rangle\right.$. This amounts to separately averaging the diffusive trajectories connecting $S_{a}$ to $S_{0}$, and $S_{b}$ to $S_{0}$, relying on energy rather than spatial separation. Then one obtains

$$
\begin{aligned}
\left\langle\left\langle(\mathcal{A})_{Q}\right\rangle\right\rangle= & 2 n_{F}(\varepsilon-e V)\left(\pi \rho_{N}\right)^{2} \tau^{8} \\
& \times\left\{P_{\beta, \gamma_{1}} \tilde{P}_{c_{1}, c_{2}} P_{\gamma_{2}, \alpha}\right\} \sin \left(\varphi_{a}+\varphi_{b}\right) .
\end{aligned}
$$

with

$$
\begin{gathered}
P_{\beta, \gamma_{1}} \equiv\left\langle\left\langle g_{\gamma_{1}, \beta}^{11}(\varepsilon-e V) g_{\beta, \gamma_{1}}^{22}(\varepsilon+e V)\right\rangle\right\rangle, \\
P_{\gamma_{2}, \alpha} \equiv\left\langle\left\langle g_{\gamma_{2}, \alpha}^{11}(\varepsilon+e V) g_{\alpha, \gamma_{2}}^{22}(\varepsilon-e V)\right\rangle\right\rangle, \\
\tilde{P}_{c_{1}, c_{2}} \equiv\left\langle\left\langle g_{c_{1}, c_{2}}^{12}(\varepsilon-e V) g_{c_{2}, c_{1}}^{21}(\varepsilon+e V)\right\rangle\right\rangle,
\end{gathered}
$$

and where the characteristic phase dependence of the quartet mode stems from the four involved Andreev reflections, one at $S_{a}$, one at $S_{b}$, and two at $S_{0}$. A product of three probabilities appear: $P_{\gamma_{2}, \alpha}$ for electron-hole (Andreev pair) diffusion from $S_{a}$ to $S_{0}, \widetilde{P}_{c_{1}, c_{2}}$ for the anomalous diffusion inside $S_{0}$, and $P_{\beta, \gamma_{1}}$ for the Andreev pair diffusion from $S_{0}$ to $S_{b}$. $\tilde{P}_{c_{1}, c_{2}}$ tracks the probability of two Andreev reflections at $S_{0}$. The Andreev pair diffusion modes $P_{i j}=P\left(R_{i j}, \omega\right)$ showing out in Eq. (A6) are obtained by a summation of the ladder diagrams [36], standard in the diffusion problem.

The diffusion probability on a distance $R$ is $P_{0}(R, \omega, V)=$ $\left\langle\left\langle g^{11, A}(R, \omega-e V) g^{22, A}(R, \omega+e V)\right\rangle\right\rangle$, and its space Fourier transform is proportional to

$$
P_{0}(\mathbf{q}, \omega, V) \sim \frac{1}{w\left(i \omega+D q^{2}\right)} .
$$

Importantly, $P_{0}(\mathbf{q}, \omega, V)$ has no dependence on $V$ in the $V \ll$ $\Delta$ limit. Most importantly, the quartet current appears even if the voltage is larger than the Thouless energy.

The above principle for the quartet current calculation can be benchmarked on the simpler case of an $S N S$ junction at equilibrium. One considers the dc Josephson current in a $S_{a} I N I S_{b}$ junction and evaluates it on the same line as above by a expansion of the current to fourth order in the transparencies.
Then the Fourier transform of the diffusion probability associated with the Andreev pair modes $\left\langle\left\langle g_{\gamma_{1}, \beta}^{11, A}(\omega-e V) g_{\beta, \gamma_{1}}^{22, A}(\omega+\right.\right.$ $e V)\rangle\rangle$ in $N$ is also given by $P_{0}(\mathbf{q}, \omega, V)$. For comparison, in a $N_{a} I N I N_{b}$ junction with mode $\left\langle\left\langle g_{\gamma_{1}, \beta}^{11, A}(\omega-e V) g_{\beta, \gamma_{1}}^{11, R}(\omega+\right.\right.$ $e V)\rangle\rangle$, the diffusion probability is $P_{0}(\mathbf{q}, 0, V)$, thus without the $i \omega$ factor in the denominator of Eq. (A10).

Depending on the diffusion taking place in $N$ or $S$, this results after integration over $q$,

$$
\begin{aligned}
& P_{0 N}(R, \varepsilon) \sim 2 \pi \rho_{N} \frac{1}{2 D R} \exp \left(-\sqrt{\frac{\varepsilon}{E_{\mathrm{Th}}}}\right) \cos \left(\sqrt{\frac{\varepsilon}{E_{\mathrm{Th}}}}\right), \\
& \tilde{P}_{0 S}(R, \varepsilon) \sim 2 \pi \rho_{N} \frac{1}{2 D R} \exp \left(-\frac{R}{\xi}\right),
\end{aligned}
$$

where the Thouless energy for a junction of length $R$ is $E_{\mathrm{Th}}=$ $\frac{\hbar D}{R^{2}}$. Notice that $\tilde{P}_{0 S}$ decays on the effective coherence length in $S_{0}$ (taking into account disorder) [37].

The quartet current calculation follows from Eqs. (A3)(A6):

$$
\begin{aligned}
I_{\mathrm{tot}} \sim & \frac{2 e}{h} \mathcal{N} \frac{\tau^{8}}{w^{2}} \int d \omega P_{\beta, \gamma_{1}}(\omega) P_{\gamma_{2}, \alpha}(\omega) \\
& \times \int_{\mathcal{S}_{c}} \frac{d^{2} r}{\xi^{2}} \tilde{P}_{c_{1}, c_{2}}(\omega)(\delta \mathcal{V})^{3} \sin \left(\varphi_{a}+\varphi_{b}\right),
\end{aligned}
$$

where $\mathcal{N}$ is the average number of channels due to integration on one of the interfaces of $S_{a, b}$. The integration volume $\delta \mathcal{V} \sim \lambda_{F} l_{e}^{2}$ accounts for the absorbing boundary conditions for diffusion in the reservoirs [36] ( $\lambda_{F}$ is the Fermi wavelength). Integration over the surface of $S_{0}$ accounts for the range $\xi$ of the Andreev reflection and yields a total factor $\frac{\xi}{w l_{e}}$ for the integrated Andreev probability in $S_{0}$. Integration over frequency yields the factor $E_{\mathrm{Th}}$, and each diffusion probability contributes by a factor $\frac{l_{e}}{w L}$. One finally obtains

$$
e I_{Q} \sim-G_{\mathrm{CAR}} E_{\mathrm{Th}} \sin \left(\varphi_{a}+\varphi_{b}\right) .
$$

A yet unknown prefactor has to be added in Eq. (2), which is expected to be of the same order as that involved in the case of a $S N S$ junction.

The conductance $G_{\mathrm{CAR}}$ refers to the CAR in a $N_{a} N S_{0} N N_{b}$ hybrid structure. The maximum quartet current is thus naturally obtained by multiplying the CAR conductance by the Thouless energy that sets the coherence of Andreev pairs on both branches $a, b$ of the bijunction.

The CAR conductance is evaluated from above,

$$
G_{\mathrm{CAR}} \sim \frac{2 e^{2}}{h} \mathcal{N}\left(\frac{\tau}{w}\right)^{8}\left(\frac{l_{e}}{L}\right)^{2} \frac{\xi}{l_{e}},
$$

where the ratio $\frac{\tau}{w}$ is taken from the rather good experimental conductance $T \sim 0.3$.
[1] K. Likharev, Dynamics of Josephson Junctions and Circuits (Gordon and Breach, New York, 1986).

[2] A. F. Andreev, Sov. Phys. JETP 19, 1228 (1964).

[3] T. M. Klapwijk, G. E. Blonder, and M. Tinkham, Physica B+C 109-110, 1657 (1982).

[4] M. Octavio, M. Tinkham, G. E. Blonder, and T. M. Klapwijk, Phys. Rev. B 27, 6739 (1983).
[5] H. Courtois, P. Gandit, B. Pannetier, and D. Mailly, Superlattices Microstruct. 25, 721 (1999).

[6] J. M. Byers and M. E. Flatté, Phys. Rev. Lett. 74, 306 (1995).

[7] M. P. Anantram and S. Datta, Phys. Rev. B 53, 16390 (1996).

[8] J. Torrès and T. Martin, Eur. Phys. J. B 12, 319 (1999).

[9] G. Deutscher and D. Feinberg, Appl. Phys. Lett. 76, 487 (2000). 
[10] P. Recher, E. V. Sukhorukov, and D. Loss, Phys. Rev. B 63, 165314 (2001).

[11] D. Beckmann, H. B. Weber, and H. v. Löhneysen, Phys. Rev. Lett. 93, 197003 (2004).

[12] P. Cadden-Zimansky and V. Chandrasekhar, Phys. Rev. Lett. 97, 237003 (2006).

[13] L. Hofstetter, J. Csonka, S. Nygård, and C. Schönenberger, Nature (London) 461, 960 (2009).

[14] L. G. Herrmann, F. Portier, P. Roche, A. L. Yeyati, T. Kontos, and C. Strunk, Phys. Rev. Lett. 104, 026801 (2010).

[15] A. Das, Y. Ronen, M. Heiblum, D. Mahalu, A. V. Kretinin, and H. Shtrikman, Nat. Commun. 3, 1165 (2012).

[16] S. Duhot, F. Lefloch, and M. Houzet, Phys. Rev. Lett. 102, 086804 (2009).

[17] M. Houzet and P. Samuelsson, Phys. Rev. B 82, 060517 (2010).

[18] B. Kaviraj, O. Coupiac, H. Courtois, and F. Lefloch, Phys. Rev. Lett. 107, 077005 (2011).

[19] F. Giazotto and F. Taddei, Phys. Rev. B 84, 214502 (2011).

[20] A. V. Galaktionov, A. D. Zaikin, and L. S. Kuzmin, Phys. Rev. B 85, 224523 (2012).

[21] J. C. Cuevas and H. Pothier, Phys. Rev. B 75, 174513 (2007).

[22] A. Freyn, B. Douçot, D. Feinberg, and R. Mélin, Phys. Rev. Lett. 106, 257005 (2011).

[23] T. Jonckheere, J. Rech, T. Martin, B. Douçot, D. Feinberg, and R. Mélin, Phys. Rev. B 87, 214501 (2013).

[24] N. M. Chtchelkatchev, T. I. Baturina, A. Glatz, and V. M. Vinokur, Phys. Rev. B 82, 024526 (2010).
[25] P. Dubos, H. Courtois, O. Buisson, and B. Pannetier, Phys. Rev. Lett. 87, 206801 (2001).

[26] C. Hoffmann, F. Lefloch, M. Sanquer, and B. Pannetier, Phys. Rev. B 70, 180503 (2004).

[27] A. H. Pfeffer, B. Kaviraj, O. Coupiac, and F. Lefloch, Rev. Sci. Instrum. 83, 115107 (2012).

[28] F. Pierre, A. Anthore, H. Pothier, C. Urbina, and D. Esteve, Phys. Rev. Lett. 86, 1078 (2001).

[29] H. Courtois, M. Meschke, J. T. Peltonen, and J. P. Pekola, Phys. Rev. Lett. 101, 067002 (2008).

[30] M. A. H. Nerenberg, J. A. Blackburn, and D. W. Jillie, Phys. Rev. B 21, 118 (1980).

[31] D. W. Jillie, M. A. H. Nerenberg, and J. A. Blackburn, Phys. Rev. B 21, 125 (1980).

[32] A. F. Volkov and H. Takayanagi, Phys. Rev. Lett. 76, 4026 (1996).

[33] N. Argaman, Superlattices Microstruct. 25, 861 (1999).

[34] K. W. Lehnert, N. Argaman, H.-R. Blank, K. C. Wong, S. J. Allen, E. L. Hu, and H. Kroemer, Phys. Rev. Lett. 82, 1265 (1999).

[35] J. C. Cuevas, A. Martín-Rodero, and A. Levy Yeyati, Phys. Rev. B 54, 7366 (1996).

[36] E. Akkermans and G. Montambaux, Mesoscopics Physics of Electrons and Photons (Cambridge University Press, Cambridge, UK, 2007).

[37] D. Feinberg, Eur. Phys. J. B 36, 419 (2003). 